\title{
Efficient Detection of Native and Bypass Coronary Ostia in Cardiac CT Volumes: Anatomical vs. Pathological Structures
}

\author{
Yefeng Zheng ${ }^{1}$, Huseyin Tek ${ }^{1}$, Gareth Funka-Lea ${ }^{1}$, \\ S. Kevin Zhou ${ }^{1}$, Fernando Vega-Higuera ${ }^{2}$, and Dorin Comaniciu ${ }^{1}$ \\ 1 Image Analytics \& Informatics, Siemens Corporate Research, Princeton, NJ, USA \\ 2 Computed Tomography, Healthcare Sector, Siemens AG, Forchheim, Germany \\ yefeng.zheng@siemens. com
}

\begin{abstract}
Cardiac computed tomography (CT) is the primary noninvasive imaging modality to diagnose coronary artery disease. Though various methods have been proposed for coronary artery segmentation, most rely on at least one user click to provide a seed point for initialization. Automatic detection of the coronary ostia (where coronaries originate from the aorta), including both the native coronary ostia and graft ostia of the bypass coronaries, can make the whole coronary exam workflow fully automatic, therefore increasing a physician's throughput. Anatomical structures (native coronary ostia) and pathological structures (graft ostia) often require significantly different detection methods. The native coronary ostia are well constrained by the surrounding structures, therefore are detected as a global object. Detecting the graft ostia is far more difficult due to the large variation in graft position. A new searching strategy is proposed to efficiently guide the focus of analysis and, at the same time, reduce the false positive detections. Since the bypass coronaries are grafted on the ascending aorta surface, the ascending aorta is first segmented to constrain the search. The quantitative prior distribution of the graft ostia on the aorta surface is learned from a training set to significantly reduce the searching space further. Efficient local image features are extracted around each candidate point on the aorta surface to train a detector. The proposed method is computationally efficient, taking about 0.40 seconds to detect both native and graft ostia in a volume with around $512 \times 512 \times 200$ voxels.
\end{abstract}

\section{Introduction}

Cardiovascular disease (CVD) is the first leading cause of death in the United States and coronary stenosis (narrowing of the vessel) is the most common CVD [1. If the stenosis is too severe and medical therapy does not help, an artery or vein from elsewhere in the patient's body is often harvested and grafted to the coronary arteries to bypass the stenosis (see Fig. 11). Cardiac computed tomography $(\mathrm{CT})$ is the primary non-invasive imaging modality to diagnose coronary stenosis thanks to its superior image resolution. To facilitate the diagnosis, it

G. Fichtinger, A. Martel, and T. Peters (Eds.): MICCAI 2011, Part III, LNCS 6893, pp. 403-410, 2011. (C) Springer-Verlag Berlin Heidelberg 2011 

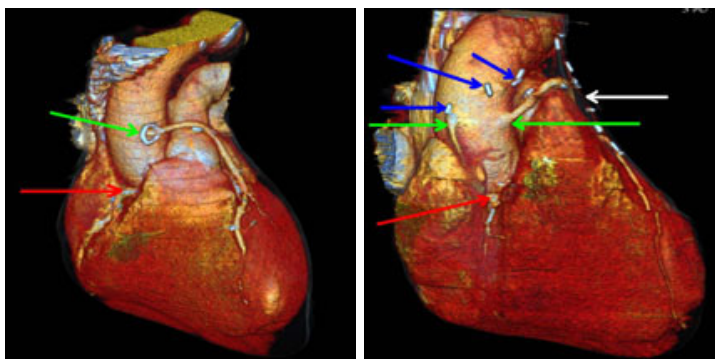

Fig. 1. 3D coronary volumetric visualization for two datasets, showing the right coronary ostia (red arrows), graft ostia of bypass coronaries (green arrows), left internal thoracic artery bypass (white arrow), and surgical clips (blue arrows). The left coronary ostium is not visible due to occlusion of the right ventricle.

is very important to develop a robust system that can efficiently extract the coronary artery centerline, followed by vessel lumen segmentation, to provide quantification of the coronary stenosis (i.e., measuring the percentage of the lumen area blocked by plaques). Though various methods have been proposed for coronary artery segmentation, most rely on at least one user click to provide a seed point to initialize the centerline extraction [2. Coronary segmentation is a time consuming task. It is desirable to perform it automatically in a preprocessing step, therefore, when a physician starts the exam, the segmentation is readily available. Automatic detection of the coronary ostia opens the potential to make the whole workflow fully automatic, therefore increasing a physician's throughput.

Almost all previous methods on native coronary ostium detection start with an explicit segmentation of the aorta first. The coronary artery is detected as a tubular structure attached to the aorta and the position of the attachment is taken as a detected ostium. Many methods require at least one user click inside the aorta to initialize the aorta segmentation, even though one click is not sufficient to guarantee good detection of the ostia [3]. Recently a few automatic coronary ostium detection methods have been proposed. Wang and Smedby 4] used a Hough transform based 2D circle detection algorithm to detect and trace the ascending aorta. The largest connected components on each side of the aortic root were picked as the left and right coronary arteries. The method proposed by Tek et al. [5] also performed automatic aorta segmentation. They then tried to trace coronary centerlines from the aorta surface in order to detect coronary ostia. Since the computationally expensive centerline tracing algorithm needed to be run on the whole aorta surface, their approach was slow. Some native coronary ostium detection methods can be extended to detect graft ostia. However, the performance is either not reported [5]4] or poor [3].

As a prerequisite for all the following coronary analysis procedures, coronary ostium detection have to be fast and robust. In this paper, we propose an efficient method to automatically detect both native coronary ostia and graft ostia of bypass coronaries. Anatomical structures (native coronary ostia) and pathological 
structures (graft ostia) often require significantly different detection methods. Since the native coronary ostia are well constrained by the neighboring global structures, we can detect them directly without explicit segmentation of the aorta as done in the previous work [345]. As a pathological structure, a graft ostium has far less constraint in its exact position (see Fig. 11). The number of bypass coronaries also varies quite a lot, from one up to four. To address these challenges, a different searching strategy is proposed to efficiently guide the focus of analysis and, at the same time, reduce the false positive detections. We first segment the ascending aorta and constrain the graft ostium detection onto the aorta surface where a bypass coronary is anastomosed 1 The quantitative prior distribution of the graft ostia on the aorta surface is learned from a training set to significantly reduce the searching space further. Efficient local image features are extracted around each candidate point on the aorta surface to train a detector. The proposed approach is computationally efficient, taking about 0.40 seconds to detect both native and graft ostia.

\section{Native Coronary Ostium Detection}

As an anatomical structure, the native coronary ostium has strong constraints that facilitate the automatic detection. For example, a patient contains two (and only two) native coronary ostia and the left/right ostium is located on the left/right cusp of the aortic root, respectively. For chronic total occlusion patients, the artery originated from a coronary ostium may be completely obstructed thus not visible in a cardiac CT volume. However, the correct position of the native ostium can still be inferred from the surrounding tissues. In this paper, we propose an efficient (taking a fraction of a second) and robust method to detect native coronary ostia as a global object. Different to the previous methods [354, no explicit aorta segmentation is necessary. There are two coronary ostia. Though it is possible to detect each independently, the detection results may be inconsistent in geometry. It also wastes computation power by ignoring the strong geometric constraint between the ostia. We propose to detect them as a global object (a bounding box) comprised with both coronary ostia. From the position, orientation, and scale of this global object, we can infer the position of individual ostium. As shown in Fig. 2, the bounding box is defined as a cube with one side aligned with the direction connecting the left and right coronary ostia, and the second side aligned with the aortic root centerline. The coronary ostia are located at the center of two opposite faces of the cube. The marginal space learning (MSL) method [6] is used to detect the bounding box. MSL is an efficient and robust method to detect a 3D anatomical structure in medical images based on discriminative machine learning techniques. Due to the space limit, we would like to refer readers to [6] for more details of MSL.

\footnotetext{
${ }^{1}$ We do not need to detect the the left/right internal thoracic artery (LITA/RITA) ostium of the in-situ LITA/RITA graft since the standard cardiac CT protocol does not cover the ostium on the subclavian artery (as shown in Fig. 1).
} 

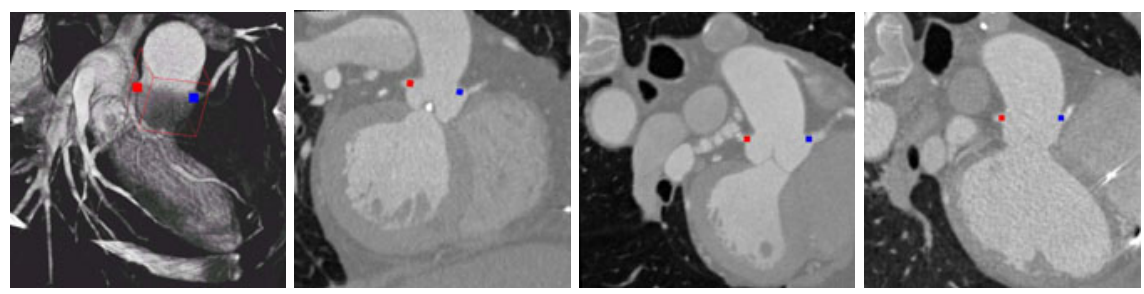

Fig. 2. Native coronary ostium detection. First column shows the detected bounding box of the coronary ostia. The right columns show the detection results on three datasets (red dot for the left ostium and blue for the right).

\section{Graft Ostium Detection for Bypass Coronaries}

\subsection{Learning Prior Distribution of Graft Ostia}

The bypass coronary arteries are attached on the ascending aorta. We can reduce the detection errors by constraining the searching of graft ostia onto the aorta surface, which is segmented automatically using the method proposed in [7. Though distribution of the graft ostia on the aorta surface is widely spread, a bypass coronary is always grafted on the anterior surface of the ascending aorta (as shown in Fig. 31) since if it is grafted in a posterior position, a physician needs to wind the bypass coronary around the aorta to bring it to the heart surface, which introduces unnecessary risks associated with the pressure caused by motion of the aorta and heart chambers. However, such observation is vague domain knowledge and a quantitative prior distribution of the graft ostia is desired to optimally use this domain knowledge.

To learn the prior distribution probability of graft ostia, we need to establish the point correspondence of the aorta surface meshes across different volumes. The length of the aorta captured in a volume varies significantly, presenting a big challenge to volume registration and surface mesh registration methods. Furthermore, the aorta has a simple tubular structure with a smooth surface. It is difficult to identify a fiducial point to establish the correspondence. In this paper, we introduce a consistent resampling method to establish the mesh correspondence by exploiting the convenience of the tubular shape of the aorta. First, we uniformly resample the aorta centerline under a certain resolution (1 $\mathrm{mm}$ in our case), starting from the aortic root and extending to the aortic arch. In this way, we establish the correspondence of the aorta centerline after resampling, even though its length varies. At each resampled centerline point, we calculate the 2D intersection of the aorta mesh and the plane perpendicular to the centerline. The 2D intersection contour is close to an ellipse, which is then uniformly resampled to 96 points with a clock-wise ordering. To establish a consistent ordering of these 96 points, the point with the smallest $x$-coordinate position is selected as the first point. The number of sampling points (96 in this case) is selected such that the distance between neighboring resampled points is close to $1 \mathrm{~mm}$. Thus, we establish the point correspondence of the $2 \mathrm{D}$ mesh 

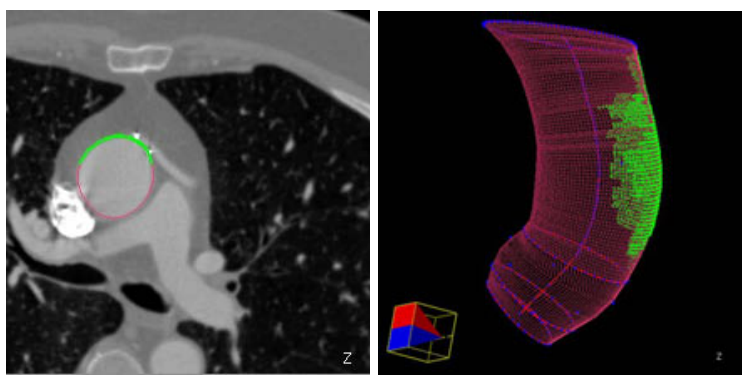

Fig. 3. Constrained searching range of the graft ostia of bypass coronaries on the aorta surface. Left: The graft ostia are constrained to the anterior part of the ascending aorta. Right: The distribution of graft ostia on the 3D aorta surface mesh. Note: The aorta mesh is shown in red, while the green dots show the constrained searching region for the graft ostia.

intersection. After triangulating the resampled points into a surface mesh (as shown in Fig. 3), we establish good correspondence for the aorta mesh points across different volumes, therefore we can align all meshes into a common coordinate system.

Given a training set of $M$ volumes with annotated graft ostia, we use the Parzen window based method to estimate the prior distribution density. Here, the Gaussian kernel is used

$$
P_{0}(x)=\frac{1}{M} \sum_{i=1}^{M}\left[\sum_{j=1}^{N_{i}} \frac{1}{2 \pi \sigma} \exp \left(-\frac{\left\|x-x_{i}^{j}\right\|^{2}}{2 \sigma^{2}}\right)\right],
$$

where $N_{i}$ is the number of graft ostia in training volume $i, x_{i}^{j}$ is the position of $j^{\text {th }}$ graft ostium in volume $i$, and $\sigma$ is the standard deviation of the Gaussian kernel. Throughout our experiments, we set $\sigma=1 \mathrm{~mm}$ and the Gaussian distribution is truncated after $3 \sigma$.

After learning prior distribution of graft ostia from a training set, only mesh points with $P_{0}(x)>0$ (green dots in Fig. 3) are considered in the following graft ostium detection. Those points with $P_{0}(x)=0$ can be excluded. Depending on the portion of the aorta captured in a CT volume, the excluded region varies. In our datasets, on average $80 \%$ of the aorta surface can be safely excluded. The prior probability $P_{0}(x)$ at each mesh point can be further used to weight the detection score during graft ostium detection, as discussed in the next section.

\subsection{Graft Ostium Detection}

We train a classifier that can tell us whether a mesh point is a graft ostium or not. Since the aorta mesh is so densely resampled, it often contains more than 10,000 points. After excluding all points with $P_{0}(x)=0$ using the prior distribution, we still need to classify a relatively large number of samples $(\sim 2,000$ points $)$. Therefore, the image features used for classification need to be efficient. In this 
paper, we use the steerable features as proposed in [6]. As suggested by [6], we use a regular sampling pattern with $5 \times 5 \times 5$ sampling points. The sampling pattern is aligned with the aorta surface normal at the mesh point under testing. At each sampling point, we extract 24 local image features as specified in [6]. In total, we get $5 \times 5 \times 5 \times 24=3000$ features. The extracted features are used to train a boosting classifier [8], which outputs a classification score in the range of $[0,1]$. The higher the score is, the more likely the candidate is a graft ostium.

We want to integrate the prior distribution of a graft ostium with the classification score to further improve the detection accuracy. Suppose $E=1$ represents the event that a graft ostium presents and $F(x)$ is the feature vector extracted at mesh point $x$. We approximate the posterior probability $P(E=1 \mid x, F(x))$ of observing a graft ostium at position $x$ as

$$
P(E=1 \mid x, F(x)) \propto P(E=1 \mid x) P(E=1 \mid F(x)),
$$

where $P(E=1 \mid x)$ is the prior probability of observing a graft ostium at $x$ and $P(E=1 \mid F(x))$ is the conditional probability given feature vector $F(x)$. Here, $P(E=1 \mid F(x))$ is replaced by the classification score, which is a good approximate as shown in [8]. In practice, we normalize $P(E=1 \mid x)$ with its maximum value

$$
P(E=1 \mid x, F(x))=\frac{P(E=1 \mid x)}{\max _{x}\{P(E=1 \mid x)\}} P(E=1 \mid F(x)) .
$$

After classification, all points with an estimated posterior probability larger than 0.5 are kept. We then perform clustering analysis on the aorta surface. The cluster center is output as a detected graft ostium. Additional validation (e.g., coronary centerline tracing from detected ostium) can further reduce the false positives.

\section{Experiments}

To evaluate the accuracy of the native coronary ostium detection, we collected and annotated 1360 datasets. A four-fold cross-validation is performed for evaluation. The Euclidean distance from the detected left coronary ostium to the corresponding ground truth has a mean of $1.66 \mathrm{~mm}$ (standard deviation of 1.15 $\mathrm{mm}$ ) and a median of $1.45 \mathrm{~mm}$. The corresponding errors of the right coronary ostium are $1.73 \mathrm{~mm}$ for the mean (standard deviation of $1.71 \mathrm{~mm}$ ) and 1.31 $\mathrm{mm}$ for the median. The maximum detection error of the left and right coronary ostia is $9.05 \mathrm{~mm}$. Only about $0.8 \%$ of datasets have a detection error larger than $5 \mathrm{~mm}$ (which is approximately the diameter of the coronary arteries around the ostia) and are treated as failures. Fig. 2 shows some examples of the detected native coronary ostia. We also compare with the method proposed by Tek et al. 5]. Their success rate on these datasets is about $90 \%$, which is significantly lower than ours.

Among the 1360 datasets, 224 have bypass coronaries with a total of $367 \mathrm{graft}$ ostia. Again, a four-fold cross-validation is performed for evaluation. It is not trivial to evaluate the detection accuracy of the graft ostia since, as pathological 
structures, the number of graft ostia varies. We may have multiple detections around a true graft ostium, or multiple true graft ostia may be merged into one detection. We use the Hungarian algorithm to establish the one-to-one correspondence between the detection graft ostia and the ground truth [9]. A true ostium is claimed to be detected correctly, if its distance to the matched detection has a distance less than a threshold. Otherwise, it is mis-detected. If a detection has a distance larger than a threshold to the matched ground truth, it is claimed to be a false positive. Similar to native coronary ostium detection evaluation, we set the threshold to $5 \mathrm{~mm}$ since it is the approximate diameter of a coronary around its ostium.

Using the prior distribution on the anterior ascending aorta, we detect 351 graft ostia (95.6\% detection rate) with only about 0.17 false positives per volume (row Constrained Search on Aorta of Table1). The false positives are mainly caused by surgical clips left on the aorta surface (see Fig. 1). The mean distance of the correct detections to the ground truth is $1.61 \mathrm{~mm}$. A few detection examples are shown in Fig. 4. For comparison, if we perform detection on the whole ascending aorta without using the prior distribution learned from the training set, we get 3.74 false positives/volume at the same detection rate (row Search on Whole Aorta of Table 1). We also tried to detect graft ostia using the method presented in Section 2, which is effective to detect the anatomical native coronary ostia. As shown by the row Search in Whole Volume of Table 1, the result is poor with a lower detection rate $(81.4 \%)$ and a significant higher false positive rate (10.25 dections/volume).

Our approach compares favorably to the state-of-the-art. For example, Hennemuth et al. 3 reported a detection rate of $57 \%$ for native coronary ostia and $27 \%$ for graft ostia on 61 datasets. The success rate of Wang and Smedby's method 4] for coronary ostia detection is only about $80 \%$ on 16 datasets.
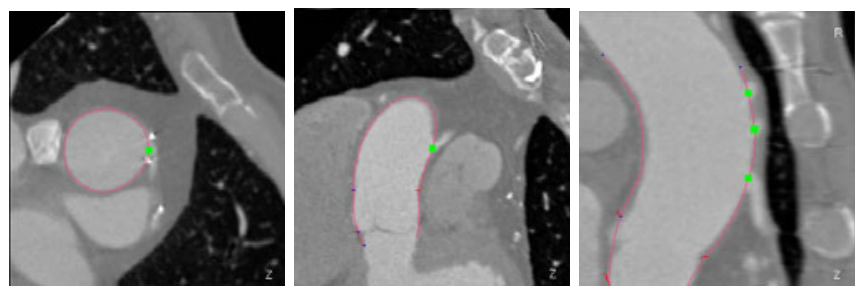

Fig. 4. Detection results for graft ostia of bypass coronaries on three datasets. The red contour shows the segmented ascending aorta and the green dots show the detected graft ostia. Note: The last dataset has three bypass coronaries.

Table 1. Comparison of graft ostium detection accuracy on 224 datasets with a total of 367 true graft ostia

\begin{tabular}{|c|c|c|c|}
\hline & \# Detections & Detection Rate & False Positives/Volume \\
\hline Search in Whole Volume & 307 & $81.4 \%$ & 10.25 \\
\hline Search on Whole Aorta & 352 & $95.9 \%$ & 3.74 \\
\hline Constrained Search on Aorta & 351 & $95.6 \%$ & 0.17 \\
\hline
\end{tabular}


On average, the proposed method takes only 0.40 seconds to process one volume on a computer with $2.33 \mathrm{GHz}$ quad-core processors and $3 \mathrm{~GB}$ memory. It spends $0.09 \mathrm{~s}$ for image preprocessing, $0.10 \mathrm{~s}$ to detect the native coronary ostia, $0.09 \mathrm{~s}$ to segment the ascending aorta, and $0.12 \mathrm{~s}$ to detect graft coronary ostia on the aorta surface. Our approach is significantly faster than the previous methods, e.g., $6 \mathrm{~s}$ reported by [5] and $88.8 \mathrm{~s}$ reported by [4].

\section{Conclusion}

In this paper, we proposed an efficient method to automatically detect both native coronary ostia and graft ostia of bypass coronaries, using totally different treatments of the anatomical structures (native coronaries) and pathological structures (bypass coronaries). It is an important step forward to make the whole coronary analysis workflow fully automatic, which can increase the throughput of a physician and reduce the inter-user variability.

\section{References}

1. Lloyd-Jones, D., Adams, R., Carnethon, M., et al.: Heart disease and stroke statistics - 2009 update. Circulation 119(3), 21-181 (2009)

2. Metz, C., Schaap, M., van Walsum, T., van der Giessen, A., Weustink, A., Mollet, N., Krestin, G., Niessen, W.: 3D segmentation in the clinic: A grand challenge II coronary artery tracking. In: Proc. MICCAI Workshop on 3D Segmentation in the Clinic: A Grand Challenge (2008)

3. Hennemuth, A., Boskamp, T., Fritz, D., Kühnel, C., Bock, S., Rinck, D., Scheuering, M., Peitgen, H.O.: One-click coronary tree segmentation in CT angiographic images. Computer Assisted Radiology and Surgery, 317-321 (2005)

4. Wang, C., Smedby, O.: An automatic seeding method for coronary artery segmentation and skeletonization in CTA. The Insight Journal (2008)

5. Tek, H., Gulsun, M.A., Laguitton, S., Grady, L., Lesage, D., Funka-Lea, G.: Automatic coronary tree modeling. The Insight Journal (2008)

6. Zheng, Y., Barbu, A., Georgescu, B., Scheuering, M., Comaniciu, D.: Four-chamber heart modeling and automatic segmentation for 3D cardiac CT volumes using marginal space learning and steerable features. IEEE Trans. Medical Imaging 27(11), 1668-1681 (2008)

7. Zheng, Y., John, M., Liao, R., Boese, J., Kirschstein, U., Georgescu, B., Zhou, S.K., Kempfert, J., Walther, T., Brockmann, G., Comaniciu, D.: Automatic aorta segmentation and valve landmark detection in C-arm CT: Application to aortic valve implantation. In: Jiang, T., Navab, N., Pluim, J.P.W., Viergever, M.A. (eds.) MICCAI 2010. LNCS, vol. 6361, pp. 476-483. Springer, Heidelberg (2010)

8. Tu, Z.: Probabilistic boosting-tree: Learning discriminative methods for classification, recognition, and clustering. In: Proc. Int'l. Conf. Computer Vision, pp. 1589 1596 (2005)

9. Liu, G., Haralick, R.M.: Optimal matching problem in detection and recognition performance evaluation. Pattern Recognition 35(3), 2125-2139 (2002) 